\title{
Proliferation and maturation of intratumoral blood vessels in women with malignant ovarian tumors assessed with cancer stem cells marker nestin and platelet derived growth factor PDGF-B
}

\author{
Sylwia Czekierdowska, Norbert Stachowicz, Mieczysław Chróściel, Artur Czekierdowski
}

Department of Gynecological Oncology and Gynecology, Medical University of Lublin, Poland

\begin{abstract}
Objectives: Platelet-derived growth factor B (PDGF-B) and nestin have been suggested to be useful in the assessment of neoangiogenesis in malignant ovarian masses. We aimed to investigate a possible association of these markers with newly formed microcapillaries and perivascular cells in ovarian tumors.

Material and methods: Microvessel density (MVD) and pericytes were studied in 82 women with ovarian neoplasms, including 7 benign cysts, 7 borderline masses, 64 epithelial ovarian cancers and 4 other malignant ovarian tumors. Immunohistochemical staining included antibodies to CD34, PDGF-B and nestin.

Results: Median values of CD34-positive and nestin-positive MVD were: 24,5 (range:17-32) and 21 (range: 12-31), respectively. No significant correlation between intratumoral CD-34 positive MVD and nestin-positive MVD was found. Benign and borderline lesions more frequently than malignant tumors displayed low or medium values of nestin-positive MVD $(p=0.01)$. Histological grading of malignant tumors was associated with nestin-positive MVD $(p=0.01)$. Nestin expression in tumor cells was not correlated with tumor grade or histological subtype. PDGF-B expression was found in tumor microvessels in $72 \%$ of cases (59/82). High expression of PDGF in pericapillary cells was strongly associated with high expression of this marker in cancer cells $(p=0.007)$. Significant correlations between PDGF-B and nestin expression in malignant tumor microvessels were also found $(p=0.04)$. Nestin and PDGF-B expressions were strongly associated with high grade tumors when compared to low grade or benign masses.

Conclusions: We conclude that the assessment of PDGF-B and nestin-positive MVD could be used to identify only highly active, angiogenic malignant ovarian masses, where tumor vasculature is formed.
\end{abstract}

Key words: ovarian cancer, microvessel desity, nestin, PDGF, pericytes

Ginekologia Polska 2017; 88, 3: 120-128

\section{INTRODUCTION}

Epithelial ovarian cancer (EOC) remains the most lethal gynecological malignancy, mainly because of late detection and rapid metastasis formation. Conventional therapies include various taxan/platinum based regimens of chemotherapy and, more recently, targeted therapies such as antivascular therapy. However, the majority of women with advanced disease will delvelop recurrence within 2 years of the initial surgical treatment. Most solid malignant tumors require angiogenesis for their growth beyond 2-3 $\mathrm{mm}$ in size [1]. Formation of microvasculature is also an essential step that allows tumor cells to enter systemic circulation of the host organism. Novel antiangiogenic cancer regimens require new studies to better understand the features of intratumoral vessels. One of the most important issues includes generation and maturation of a tumor microvascular network. Several recent studies have shown that cancer microvessels are characterized not only by abnormal pericyte coverage, but also by disturbed interactions between pericytes and endothelial cells, both 
of which may contribute to the metastasic dissemination of various cancers [2,3].

Tumor microvessels in various human cancers are structurally and functionally abnormal $[3,4]$. One of the important features of the newly formed tumor microvessels is their increased permeability to electrolytes and proteins due to different structure of the basement membrane. This feature enables malignant tumor cells to penetrate the barrier more easily than through the mature microvessels [4, 5]. Increased tumor microvessel density (MVD) could be used as a predictive indicator of poor survival in various types of cancer patients. In the last two decades MVD in various cancers has been widely studied with the use of immunohistochemistry. Vascular endothelial cells of normal tissues were commonly stained with panendothelial cells markers such as CD34, CD31 or factor VIII associated antigen, one of the essential blood clotting proteins [6]. However, these markers cannot specifically identify newly formed tumor capillaries, moreover, they usually also detect pre-existing, relatively large sized blood vessels. Because of these features, the above markers are currently not considered suitable for the sensitive analysis of tumor neoangiogenesis. Potential clinical significance of MVD analysis in women with EOC remains controversial [7]. In contrast to a variety of solid neoplasms, MVD does not seem to be a useful predictor of survival in patients with EOC. Several reasons seem to be responsible with the most important one, the heterogenic nature of microvascular network in ovarian cancer patients. Great variety of newly formed and preexisting blood vessels makes panendothelial markers like CD34 or CD31 not specific enough to show prognostic significance when tested in multivariate analysis [7].

One of the novel markers of the proliferating endotheliocytes is nestin — an intermediate filament protein, initially thought to be exclusively associated with neuro-progenitor cells [8-10]. Although nestin is expressed during early stages of normal cells development, under pathological conditions this protein could be also found in cancer stem cells, as well as in perivascular and endothelial cells suggesting its role in various malignant tumors neoangiogenesis [11, 12]. In recent years, an increased nestin expression has been reported in multiple malignant masses including CNS tumors, gastrointestinal stromal tumors, pancreatic cancer, prostate cancer, breast cancer, malignant melanomas, and thyroid tumors [13-15]. High expression of nestin correlated with aggressive growth, metastasis, and poor prognosis in these cancers. Despite extensive research published to date, not all the roles of nestin in malignant tumors growth have been well characterized. Recently, it has been suggested that nestin could serve as a novel marker of pericytes and pericyte-like mural cells and thus of vascular maturation. Remodeling of blood vessels network requires tissue-resident nestin-positive progenitor cells that represent the basic source for pericytes and smooth muscle cells. The assessment of nestin expression as a potential marker for mural pericytes was suggested to determine the mechanisms of pericytes recruitment and differentiation [13]. Pericytes are not only found close to endothelial cells, but can be searched by their localiztion to the abluminal side of the blood vessel wall in combination with the expression of various cell surface markers such as a-smooth muscle actin (a-SMA), CD146, neural glial 2 (NG2 and/or leptin receptor (LepR). Another new potential marker could be platelet-derived growth factor (PDGF) as both PDGF and its receptor-beta (PDGFR $\beta$ ) are highly expressed in pericytes [16]. Suppression of the PDGF signaling pathway leads not only to pericyte loss, but also to endothelial changes followed by capillary dilation, microaneurysm formation and rupture [17, 18]. Emerging evidence suggests that the PDGF system may play an important role in carcinogenesis and because of this several new biological agents targeting PDGF signaling pathway are currently investigated [19].

Although several studies have investigated nestin and PDGF expression in ovarian cancer, little is known about the role of these markers in a process of microvessel maturation in tumor tissues. The mechanisms responsible for pericytes recruitment, migration and regulation of tumor angiogenesis in ovarian cancer have not been fully understood. In this study we aimed to investigate the possible role of nestin as a marker of pericytes in vascular remodeling processes during tumor progression. We also aimed to determine possible associations between the microvessel count, expression of nestin and PDGF-B, and selected clinicopathological features in ovarian benign and malignant tumors.

\section{MATERIAL AND METHODS}

Primary human ovarian tumors and nonmalignant tissues were obtained from 82 women with ovarian masses who had surgery at the $1^{\text {st }}$ Department of Gynecological Oncology and Gynecology of the Medical University of Lublin between years 2009-2011. The study has been approved by the local Bioethical Committee and all participants gave their informed consent. The median age of patients was 50 years (range: 18-86 years). Among them there were 31 premenopausal (37.8\%) women, whereas 51 were postmenopausal (62.2\%). All specimens used in this study were examined and selected by an experienced pathologist to confirm the diagnosis of either benign or malignant ovarian tumors. Excluding benign masses, the proportion of malignant cells in tumor tissues was always more than $50 \%$. The histological type and grade of malignant tumors were classified according to the criteria of the World Health Organization (WHO). The stage of each cancer was established according to the International Federation 
of Gynecology and Obstetrics criteria. Final histological examination revealed 7 benign lesions ( 3 fibrothecomas, 3 ovarian strumas, 1 teratoma), 7 borderline tumors and 64 primary EOC's. In addition, the study included 4 other malignant ovarian tumors (3 granulosa cell tumors and 1 malignant Brenner tumor). The most common type of EOC was serous cancer (29 women), followed by the endometrioid cancer (19 cases). Table 1 shows selected demographic data and histological diagnosis of the studied group. Most malignant tumors were histological grade G2 (26 cases) or G3 (34 cases) and 55\% (46 cases) were FIGO stage III/IV. We also used 10 nonmalignant tissue samples from patients who had bilateral oophorectomies for suspected malignancy, but were found to have benign histology. These samples were collected from apparently normal contralateral ovaries and were used as controls for immunohistochemical studies.

Table 1. Menopausal status of the studied women and tumor characteristics including histological diagnosis. grade and clinical Federation of Gynecology and Obstetrics (FIGO staging)

\begin{tabular}{|l|c|c|}
\hline Studied group $(\mathbf{n}=\mathbf{8 2})$ & No. of patients & (\%) \\
\hline Type of lesions & & \\
\hline Benign ovarian tumors & 7 & 8.3 \\
\hline Borderline tumors & 7 & 8.3 \\
\hline Epithelial ovarian cancer & 64 & 78.5 \\
\hline Other malignant ovarian tumors & 4 & 4.7 \\
\hline Menopausal status & & \\
\hline Before menopause & 31 & 37.8 \\
\hline After menopause & 51 & 62.2 \\
\hline Histological grade & & \\
\hline 1 & 15 & 18.2 \\
\hline 2 & 26 & 31.7 \\
\hline 3 & 34 & 41.4 \\
\hline FIGO stage & & \\
\hline I & 24 & 29.2 \\
\hline II & 6 & 7.3 \\
\hline III & 42 & 51.2 \\
\hline IV & 3 & 3.6 \\
\hline Histological subtype & & \\
\hline Serous & 29 & 35.3 \\
\hline Endometrioid & 19 & 23.1 \\
\hline Mucinous & 11 & 13.4 \\
\hline Metastatic & 5 & 6.1 \\
\hline undifferentiated & 4 & 4.8 \\
\hline Anaplastic & 1 & 2.4 \\
\hline Germinal & & 1.2 \\
\hline Clear cell & & 1.2 \\
\hline & & \\
\hline
\end{tabular}

\section{Immunohistochemical studies}

Immunohistochemical analysis was performed in the paraffin wax-embedded representative tumor tissue sections fixed in 10\% neutral buffered formalin. Four-micrometer sections from formalin-fixed, paraffin-embedded tissue were mounted on silanized slides (DAKO Cytomation, Denmark). The slides were air-dried and the tissue deparaffinized and rehydrated.

The following antibodies and dilutions were used: CD34 (mouse, monoclonal IgG Class II, DAKO Cytomation; 1:50), nestin (mouse, monoclonal IgG 10c2, Santa Cruz Biotechnology 1:200) and PDGF-B (mouse monoclonal $\lg _{2 b}$ F-3; Santa Cruz Biotechnology 1:200) Following deparafinization, rehydratation and antigen retrieval with the Target Retrieval Solution (Dako Cytomation, Denmark) at $\mathrm{pH}=6.0$ three cycles of heating in a microwave oven (each of $5 \mathrm{~min}$, at $750 \mathrm{~W}$ ) were performed. Tissue sections were incubated with the primary antibody for 1 hour in room temperature. The slides were incubated with the secondary antibody conjugated with streptavidin-biotin-peroxidase complex (rabbit/mouse EnVision kit; DAKO Cytomation) and color reaction was developed using DAB (3'-3-diaminobenzidine tetrahydrochloride, DAKO) according to the manufacturer's protocol. The sections were counterstained with Mayer's hematoxylin. For each case, negative control was applied by replacing the antibody by PBS or nonimmune serum.

The average number of microvessels within selected tumor areas was assessed according to Weidner's method [6]. As recommended, sections were first examined at low magnifications ( $40 \times$ and $100 \times$ ) to identify the most vascular areas of the tumors, so called 'hotspots', i.e. the area(s) with most intense CD34 or nestin staining and apparently the highest density of microvessels. Then, as a rule, 10 fields were examined, except in a few cases where less tumor tissue was available. The counts were expressed as the average of all fields examined at high magnification (200x; HPF — high power field) with MVD defined as the mean of number of vessels/HPF). PDGF-B and nestin expression in tumor cells was assessed semi-quantitatively where both the intensity and percentage of stain-positive cells were measured. At least 10 microscopic fields in one representative tumor section were counted under $400 \times$ magnification. The intensity of staining was graded ' 0 ' for absent staining, ' 1 ' for weak, ' 2 ' for moderate, and ' 3 ' for intense immunoreactivity. The percentage of stain-positive cells was visually evaluated and cell expression was stratified as follows: low; for $<30 \%$ positive cells (weak or absent staining reaction), high $>30 \%$ of positive cells (moderate or strong staining reaction). Expression of PDGF-B in microvessels was described as negative (lack or weak staining reaction in microvessels) or positive (moderate or strong staining reaction). 


\section{Statistical analysis}

Statistical analysis was performed with the use of Statistica v. 10.0 (Statsoft, Poland). Association between categorical variables was examined by Pearson's $x^{2}$ test or Fisher's exact tests, where appropriate. Mann-Whitney nonparametric test was used to compare categorical with continuous tumor variables when there were two categories, whereas Kruskal-Wallis nonparametric test was used instead, when there were more than two categories. The criterion of statistical significance applied in all calculations was $\mathrm{p}<0.05$

\section{RESULTS}

\section{Ovarian tumor vasculature assessment} with CD34 antibody

Median CD34-positive microvessel density assessed in studied ovarian tumors was 24 (range: 5-56) per high power field. No significant differences in MVD-CD34 were found in benign, borderline and invasive tumors subgroups ( $p=0.73$ ). Histological grading and FIGO stage were not correlated with MVD-CD34 ( $p=0.4$ and $p=0.09$, respectively). Significant differences in microvessel density between histological subgroups of malignant tumors were found ( $p=0.04)$. The highest MVD-CD34 was observed in mucinous tumor type $(M D=34)$ and in non-EOC malignant tumors $(M D=39)$. In serous and endometrioid tumor types calculated median MVD-CD34 was 24/HPF. The lowest microvessel density was observed in metastatic $(M D=14)$, germinal $(M D=13)$ and clear cell $(M D=6)$ tumor types. Figure 1 shows typical example of CD34 staining in tumor microcapillaries.

\section{Nestin expression in tumor and perivascular cells}

Immunohistochemical staining revealed nestin presence in the cytoplasm of neoplastic cells and in mural cells of microcapilaries in 80 cases (97.5\%) of ovarian tumors. The

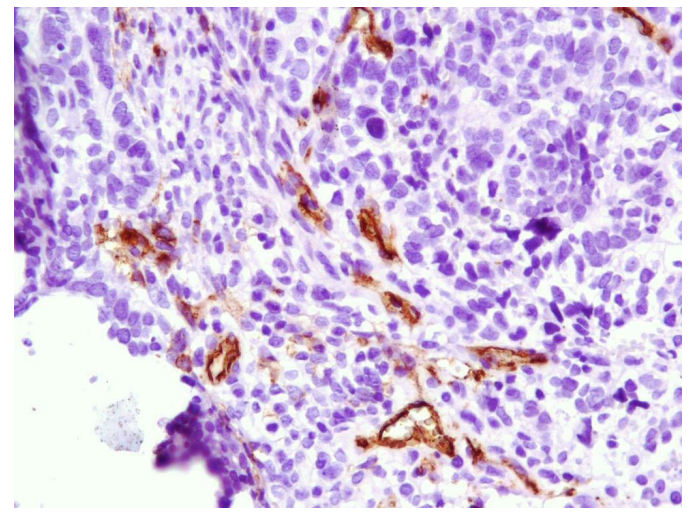

Figure 1. Example of CD34 staining in endothelial cells around tumor microvessels in ovarian serous carcinoma G3. Magnification 200x examples of nestin staining reaction are shown on Figures $2-4$. The results of nestin expression assessment in tumor cells, nestin-positive microvessel density (MVD-nestin) and their relationship with clinicopathological features are presented in Table 2. Most of the benign or borderline type

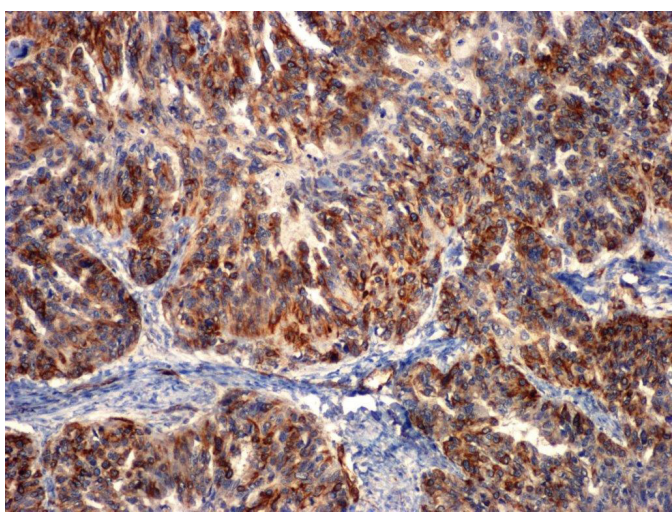

Figure 2. Example of high nestin expression in tumor cells in endometrioid cystadenocarcinoma G3. Magnification 100x

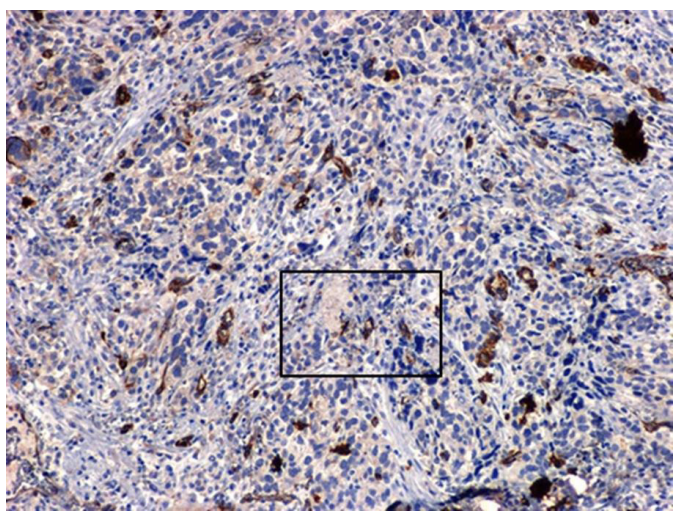

Figure 3. Example of low nestin expression in tumor cells. Staining reaction is predominantly localized in cytoplasm of endothelial cells around small microcapillaries (selected area is presented on Fig. 4). Magnification 100x

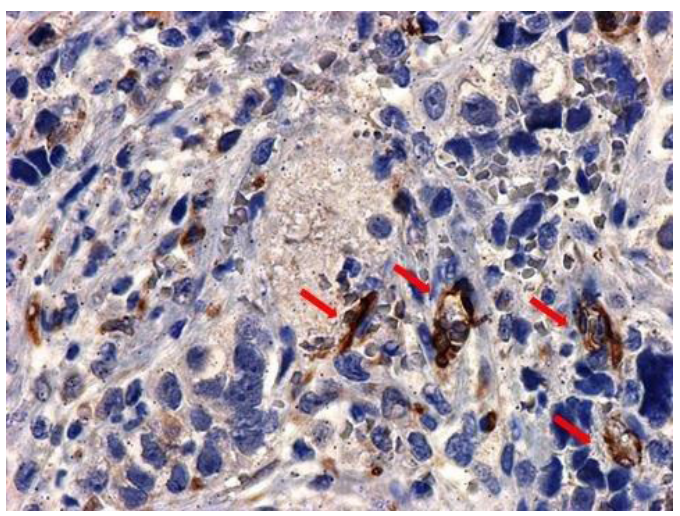

Figure 4. Nestin staining in the same sample, nestin protein is predominantly localized in the cytoplasm of endothelial cells in small microcapillaries (red arrows). Magnification 400x 
Table 2. Relationship between nestin expression in tumor cells, nestin-positive microvessel density (MVD-nestin) and clinicopathological features

\begin{tabular}{|c|c|c|c|c|c|}
\hline \multirow[b]{2}{*}{ No. of patients } & \multicolumn{3}{|c|}{ Nestin expression in tumor cells } & \multicolumn{2}{|c|}{ Microvessel density nestin-positive } \\
\hline & $\begin{array}{c}\text { Negative } \\
(n=54)\end{array}$ & $\begin{array}{l}\text { Positive } \\
(n=28)\end{array}$ & $P$ value & Median (range) & $P$ value \\
\hline \multicolumn{6}{|l|}{ Type of lesion } \\
\hline Benign ovarian tumor, $\mathrm{n}=7$ & $5(72 \%)$ & $2(28 \%)$ & \multirow{4}{*}{0.9} & $18.5(11-21)$ & \multirow{4}{*}{0.01} \\
\hline Borderline tumor, $n=7$ & $5(72)$ & $2(28 \%)$ & & $12(7-21)$ & \\
\hline Ovarian cancer, $\mathrm{n}=64$ & $42(66 \%)$ & $22(34 \%)$ & & $21(14-29)$ & \\
\hline Malignant tumor, $n=4$ & $2(50 \%)$ & $2(50 \%)$ & & $41(37-48)$ & \\
\hline \multicolumn{6}{|l|}{ Histological grading } \\
\hline $\mathrm{G} 1, \mathrm{n}=15$ & $10(67 \%)$ & $5(33 \%)$ & \multirow{3}{*}{0.9} & $12(7-22)$ & \multirow{3}{*}{0.14} \\
\hline $\mathrm{G} 2, \mathrm{n}=26$ & $16(62 \%)$ & $10(38 \%)$ & & $22(14-30)$ & \\
\hline $\mathrm{G} 3, \mathrm{n}=34$ & $23(68 \%)$ & $11(32 \%)$ & & $21(14-32$ & \\
\hline \multicolumn{6}{|l|}{ FIGO stage } \\
\hline$I+I I, n=30$ & $21(70 \%)$ & $9(30 \%)$ & \multirow{2}{*}{0.4} & $15(11-24)$ & \multirow{2}{*}{0.04} \\
\hline$I I+I V, n=45$ & $28(62 \%)$ & $17(38 \%)$ & & $24(15-35)$ & \\
\hline
\end{tabular}

FIGO — Federation of Gynecology and Obstetrics

of lesions displayed only week staining reaction in tumor cells. High nestin expression was found in 28 (34\%) cases, which included mainly women with ovarian cancer, however the differences between the groups were not statistically significant. Histological grade and FIGO stage of malignant tumors were not correlated with nestin expression in tumor cells. No significant differences in nestin expression by tumor cells according to ovarian cancer histological subtype were found.

Median density of nestin-positive microvessels in the studied tumors was 21 (range: 12-31). In women with EOC median nestin-positive MVD was MD $=21 /$ HPF (range: 14-29). The highest median MVD-nestin was observed in malignant non-EOC subgroup (42/HPF; range 37-48). The lowest MVD-nestin was found in benign and borderline tumors (12/HPF; range 7-21 and 18/HPF; range 11-21, respectively). The differences between these groups were statistically significant $(p=0.01)$. Clinical advanced tumors (FIGO III and IV) significantly more frequently displayed higher nestin-positive MVD than low FIGO stage tumors (24/HPF; range $15-35$ vs. 15 /HPF; range 11-24, respectively, $p=0.04)$. There were no significant differences in median nestin-positive MVD according to histological subtype and histopathological grade of malignant tumors. Expression of nestin in tumor cells was not associated with MVD-nestin ( $p>0.05$ ). In lesions with low expression pattern median values of nestin-positive MVD was slightly higher than in samples where staining reaction was moderate or high (22/HPF; range 13-37 vs. 19/HPF; range 12-20). No significant correlation between MVD-CD34 and nestin-positive $\operatorname{MVD}(r=0.14 ; p=0.19)$ was found.

\section{Platelet derived growth factor (PDGF-B) expression in neoplastic cells and microcapillaries}

PDGF-B antibody staining was found both in cytoplasm of neoplastic cells and in pericapillary cells (Figs. 5 and 6). No PDGF-B staining was observed in 5 cases of ovarian tumors and high expression was found in 27 (33\%) cases. Benign lesions more frequently were characterized with weak or lack of the PDGF-B expression in tumor cells (6 of 7 cases, 86\%) and EOC samples displayed more often high staining pattern in tumor cells (45 of 64 cases; 70\%). The differences between various types of lesions were statistically significant $(p=0.01)$. No significant differences were found between tumor histological type or FIGO stage when PDGF-B ex-

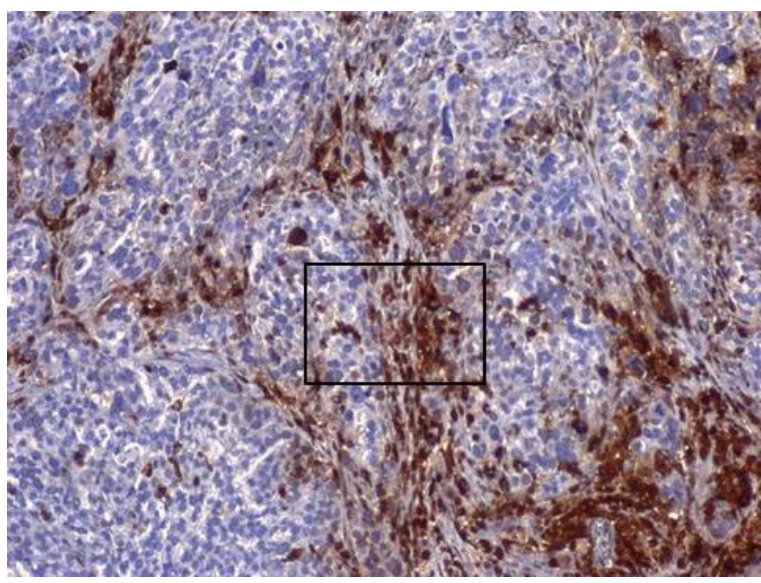

Figure 5. Example of PDGF-B staining in serous adenocarcinoma G2 (selected area is presented on Fig. 6). Magnification 100x 


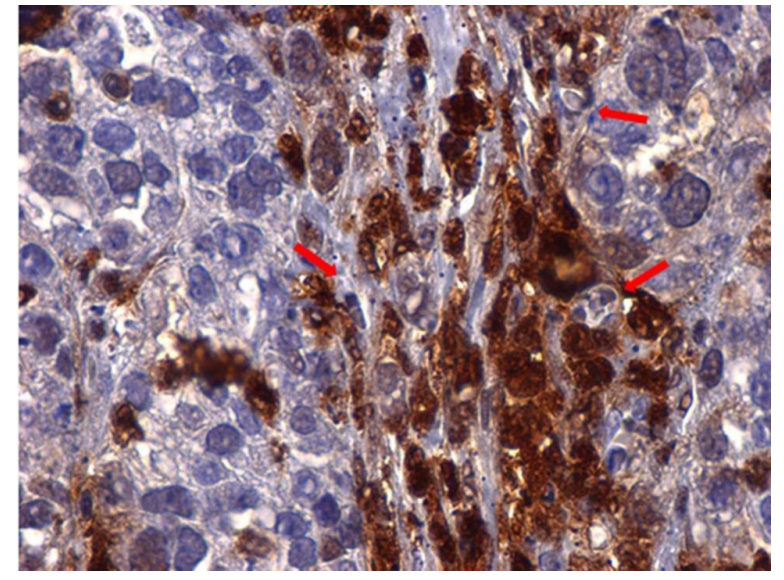

Figure 6. PDGF-B staining in serous adenocarcinoma. As shown, PDGF-B is localized in tumor cells and in cell around microvessels (probably pericytes/mural cells); red arrows point to microcapillaries with erythrocytes inside. Magnification 400x

pression was found in tumor cells ( $p>0.5$ ). Histological subtype of invasive tumors had no significant association with PDGF-B expression in tumor cells $(p>0.5)$. The results of PDGF expression assessment are shown in Table 3.

PDGF-B expression was found in tumor microvessels in $72 \%$ of cases (59 of 82). High expression of PDGF in pericapillary cells was strongly associated with high expression of this marker in neoplastic cells $(p=0.007)$. The expression pattern of PDGF-B was significantly different according to the type of lesion ( $p=0.03$ ). We have noticed that most of the benign ( 5 of 7 samples, $71 \%$ ) and all borderline tumor revealed lack or low expression pattern of PDGF-B in microcapillaries. Sig- nificant differences in PDGF-B expression in microcapillaries were correlated with histological grade of the invasive malignant tumors $(p=0.03)$. Low grade $(G 1)$ tumors more often displayed low staining pattern of PDGF-B in microvessels (11 of 15 cases, 73\%). As shown in Table 4 histological subtype or clinical stage of invasive tumors did not affect the PDGF-B expression in perivascular cells ( $p>0.05$ ). Strong associations between PDGF-B expression in tumor cells and in perivascular cells with MVD-nestin ( both $p=0.0003$ ), but not with MVD-CD34 ( $>$ > 0.05) were found. In women with cancers that had low expression pattern of tumor cells PDGF-B, median MVD-nestin was significantly lower than in tumors were the PDGF-B staining reaction was strong (16/HPF; range $7-21$ vs. 22/HPF; range 14-36, respectively). Similar

\begin{tabular}{|c|c|c|}
\hline & MVD-CD34+ & MVD-nestin+ \\
\hline Type of lesion & 0.7 & 0.01 \\
\hline Histological subtype & 0.04 & 0.3 \\
\hline Histological grading & 0.4 & 0.1 \\
\hline FIGO stage & 0.09 & 0.2 \\
\hline PDGF expression in tumor cell & 0.9 & 0.0003 \\
\hline PDGF expression in microvessels & 0.24 & 0.0003 \\
\hline Nestin expression in tumor cells & 0.09 & 0.2 \\
\hline
\end{tabular}

FIGO — Federation of Gynecology and Obstetrics; PDGF — platelet-derived growth factor

Table 3. Relationships between platelet-derived growth factor (PDGF) expression and clinicopathological features

\begin{tabular}{|c|c|c|c|c|c|c|}
\hline \multirow{3}{*}{ No. of patients } & \multicolumn{6}{|c|}{ PDGF expression } \\
\hline & \multicolumn{3}{|c|}{ In microvessels } & \multicolumn{3}{|c|}{ In tumor cell } \\
\hline & $\begin{array}{c}\text { Low } \\
(n=47)\end{array}$ & $\begin{array}{l}\text { High } \\
(n=35)\end{array}$ & $P$ value & $\begin{array}{c}\text { Low } \\
(n=28)\end{array}$ & $\begin{array}{c}\text { High } \\
(n=54)\end{array}$ & $P$ value \\
\hline \multicolumn{7}{|l|}{ Type of lesion } \\
\hline Benign tumors, $n=7$ & $5(71 \%)$ & $2(29 \%)$ & \multirow{4}{*}{0.03} & \multirow{4}{*}{$\begin{array}{c}6(86 \%) \\
3(43 \%) \\
19(30 \%) \\
0(0 \%)\end{array}$} & $1(14 \%)$ & \multirow{4}{*}{0.01} \\
\hline Borderline tumors, $n=7$ & $7(100 \%)$ & $0(0 \%)$ & & & $4(57 \%)$ & \\
\hline Ovarian cancer, $\mathrm{n}=64$ & $33(52 \%)$ & $31(48 \%)$ & & & 45 (70\%) & \\
\hline Other malignant tumors, $\mathrm{n}=4$ & $2(50 \%)$ & $2(50 \%)$ & & & $4(100 \%)$ & \\
\hline \multicolumn{7}{|l|}{ Histological grading } \\
\hline $\mathrm{G} 1, \mathrm{n}=15$ & $11(73 \%)$ & $4(27 \%)$ & \multirow{3}{*}{0.03} & \multirow{3}{*}{$\begin{array}{l}6(40 \%) \\
8(30 \%) \\
8(24 \%)\end{array}$} & $9(60 \%)$ & \multirow{3}{*}{0.5} \\
\hline $\mathrm{G} 2, \mathrm{n}=26$ & $9(36 \%)$ & $17(66 \%)$ & & & $18(70 \%)$ & \\
\hline $\mathrm{G} 3, \mathrm{n}=34$ & $20(59 \%)$ & $14(41 \%)$ & & & $26(76 \%)$ & \\
\hline \multicolumn{7}{|l|}{ FIGO stage } \\
\hline$I+I I, n=30$ & $19(53 \%)$ & $21(47 \%)$ & \multirow{2}{*}{0.15} & \multirow{2}{*}{$\begin{array}{l}11(37 \%) \\
19(63 \%)\end{array}$} & $11(24 \%)$ & \multirow{2}{*}{0.25} \\
\hline $\mathrm{II}+\mathrm{IV}, \mathrm{n}=45$ & $21(47 \%)$ & $24(53 \%)$ & & & $34(76 \%)$ & \\
\hline
\end{tabular}

FIGO - Federation of Gynecology and Obstetrics 
differences in MVD-nestin were found in tumors with low and high expression pattern PDGF-B in perivascular cells (17/HPF; range 10-22 vs. 25/HPF; range 15-38 respectively).

\section{DISCUSSION}

Most women with advanced ovarian cancer although usually sensitive to the primary chemotherapy will relapse within next few years. An improvement of the treatment outcome may be based on a higher number of the individualized therapies that will be based on the new, clinically validated predictive and prognostic markers including novel tumor angiogenesis factors. Neoangiogenesis in malignant tumors requires both endothelial cell proliferation and pericytes activation for the stabilization of microvessel sprouts. In our study we have attempted to determine the linkage between nestin, PDGF and degree of maturation of tumor vasculature in benign and malignant ovarian neoplasms. Our results confirmed previously reported observations that nestin is frequently expressed in malignant ovarian tumors [14]. Strong staining pattern of nestin was observed both in tumor cells and in mural cells of tumor microcapillaries. We have also found that nestin expression in tumor microvessels, but not in cancer cells increased with tumor grade and clinical stage of the disease. The lowest medians of nestin positive microvessels were found in benign and borderline ovarian tumors. Clinically advanced cancers more frequently displayed higher median MVD-nestin positive microvessels than low FIGO stage tumors.

Nestin has been reported to correlate with aggressive growth, metastasis, and poor prognosis in several malignancies; however, the role of nestin in ovarian cancer neoangiogenesis has not been well characterized $[8,11$, $14,15]$. Compared to MVD studies that used several panendothelial markers, only a limited number of investigations have employed nestin. Nestin could serve not only as a reliable neovascularization marker but also may reflect cancer stem cell properties in different types of malignant masses, correlating with poor prognosis. Osman et al. [20] found the expression of nestin in almost $50 \%$ of all epithelial ovarian cancers and this neurofilament protein was expressed more often (60\%) in serous type of tumors. In our study nestin expression pattern in microvessels did not correlate with nestin expression in tumor cells. Also, no association between MVD-nestin positive microvessels and MVD-CD34 stained microvessels was found. Interestingly, nestin-positive cells were identificated in the newly formed blood vessels suggesting that these cells might have been derived from nestin-positive tissue-resident multipotent stem cells. In contrast, mature vessels were either negative or only slightly positive for nestin immunostaining and mainly dispalyed CD34-positive staining pattern. Interestingly, we have found that tumors with lower number of CD34-positive microvessels more often were expressing a higher number of nestin-positive microvessels. Conversely, higher density of CD34-positive microvessels was observed in tumors with lower counts of nestin-positive MVD. This observation confirms that nestin could be a more appropriate marker to identify only highly angiogenic malignant ovarian lesions where tumor vasculature is actively formed.

The origin of nestin-positive vascular endothelial cells in tumor tissue is still controversial. As a possible mechanism, a migration of mesenchymal stem cells from the bone marrow has been proposed by Dong et al. who showed that human glioma stem/progenitor cells transdifferentiate into vascular endothelial cells in vitro and in vivo [21]. Although it is still not known whether nestin expression is restricted to angiogenic endothelial cells or to nestin expressing stem cells that are recruited to the newly formed blood vessels, our results are in line with the hypothesis that nestin could be included as a novel marker of neoangiogenesis and vascular remodeling proceses in ovarian cancer.

The mechanisms responsible for pericytes recruitment, migration and regulation of tumor angiogenesis have not been fully understood. PDGF may trigger microvessel formation by priming vascular smooth muscle cells and/or pericytes to release pro-angiogenic factors. Emerging evidence suggests that the PDGF system plays an essential role in ovarian cancer progression [22]. Still, there are only few published studies on PDGF-B expression in ovarian malignancies $[23,24]$. Our results confirmed that in women with ovarian cancer the sources of PDGF-B could be both tumor cells and endothelial cell/pericytes. We have found that expression of PDGF-B increased with the aggressiveness of tumors as assessed by tumor histological grade. Benign or borderline lesions more frequently had low expression of PDGF-B, whereas high grade and FIGO advanced stage types of ovarian cancer displayed more often strong staining pattern of this marker both in cancer cells and in microvessels. Our results are in agreement with previous findings that during vascular formation activated endothelial cells or tumor cells can secrete PDGF-B and paracrine stimulation of PDGF receptor (PDGF-R) promotes pericyte precursor cell to proliferation and migration [25]. We have found that a high ratio of PDGF-B expression in microvessels is correlated with higher density of nestin-positive microvessels but not with CD34-positive MVD. Only few reports have focused on PDGF isoforms or their receptors expression in women with ovarian cancer and to date, the role of the PDGF-B signaling pathway has not yet been explained.

Madsen et al. [23] demonstrated the presence of target for both PDGFR- $\alpha$ and PDGFR- $\beta$ in the tumor and stromal compartments of a substantial proportion of the ovarian cancer samples. They found high PDGFR- $\beta$ expression in tumor cells in $60 \%$ of clear cell adenocarcinomas, but no expression 
of PDGFR- $a$. However, in stromal cells $40 \%$ of their cases stained positive for PDGFR- $a$. In another study from the same group Madsen et al. [24] have measured serum PDGF-AA, PDGF-BB, FGF2, and VEGF concentrations in bevacizumab treated multiresistant ovarian cancer patients. The levels of these cytokines were up-regulated in ovarian cancer and were associated with stage and residual disease. More recently, Hosaka et al. [25] have demostrated that PDGF-B induces pericyte-fibroblast transition (PFT), which was significantly correlated with tumor invasion properties. Moreover, they also demontrated that the coimplantation of PFT cells with less-invasive tumor cells in mice markedly promoted tumor dissemination and invasion, leading to an increased number of circulating tumor cells and metastasis [25].

Tumor vessels are stabilized by the integration of smooth muscle cells and pericytes. Such maturation and stabilization of the new microvessels is thought to be responsible for their resistance to the drugs used in the antivascular tumor therapy [13]. Therefore, new treatment modalities are urgently needed. Targeting multiple angiogenic signaling pathways by various molecular agents including tyrosine kinase inhibitors is a promising strategy to modulate tumor vascularization, microcirculation, and effectively block the growth of angiogenesis-dependent tumors. Additionally, pericyte-targeting seems to be an effective supporting anticancer therapy. Recently, Lu et al. [26] tested the efficacy of a highly selective aptamer (AX102) against PDGF-B with or without anti-VEGF therapy (bevacizumab) in ovarian cancer models in vitro. They found that combination of two agents for endothelial (bevacizumab) and pericyte (AX102) targets significantly reduced MVD, vascular maturation as assessed by pericyte coverage fraction and proliferation index (PCNA) in their treatment group. In summary, our results demonstrate the importance of nestin and PDGF signaling in promoting vascular growth, vessel maturation and vascular remodeling in malignant ovarian tumors.

\section{CONCLUSIONS}

1. Nestin as a novel marker for mural pericytes could be used to better characterize the mechanisms of pericytes recruitment and differentiation and their relationship to clinical outcome in women with malignant ovarian tumors.

2. Ovarian cancers should be checked for alternative angiogenic pathways to look for possible new multidrug antiangiogenic treatment stategies.

\section{Acknowledgments}

This work was supported by grant \#DS119 to AC from the Medical University of Lublin. The Authors wish to thank dr Jadwiga Sierocińska-Sawa, Head of the Univeristy's Clinical Hospital No.1 Pathology Unit for her expertise in tissue samples sellection and analysis.

\section{Authors' contributions}

SC, NS, MC, AC had full access to all the data in the study and take responsibility for the integrity of the data, the accuracy of the data analysis, and the decision to submit for publication. SC, NS, AC conceived, designed and obtained funding for the study. NS and MC collected the samples and patient's data. SC performed immunohistochemical studies. SC, NS and $M C$ analyzed the data and drafted the manuscript. AC supervised the study and revised the manuscript. All authors interpreted the data, critically revised the draft for important intellectual content, and gave final approval of the manuscript to be published.

\section{REFERENCES}

1. Folkman J, Ryeom S, Folkman J, et al. What is the evidence that tumors are angiogenesis dependent? J Natl Cancer Inst. 1990; 82(1): 4-6, indexed in Pubmed: 1688381.

2. Baluk P, Morikawa S, Haskell A, et al. Abnormalities in pericytes on blood vessels and endothelial sprouts in tumors. Am J Pathol. 2002; 160(3): 985-1000, doi: 10.1016/S0002-9440(10)64920-6, indexed in Pubmed: 11891196.

3. Bergers G, Song S, Meyer-Morse N, et al. Benefits of targeting both pericytes and endothelial cells in the tumor vasculature with kinase inhibitors. J Clin Invest. 2003; 111(9): 1287-1295, doi: 10.1172/JCI17929, indexed in Pubmed: 12727920.

4. Hashizume H, Baluk P, Morikawa S, et al. Openings between defective endothelial cells explain tumor vessel leakiness. Am J Pathol. 2000; 156(4): 1363-1380, doi: 10.1016/S0002-9440(10)65006-7, indexed in Pubmed: 10751361.

5. Jain RK. Normalization of tumor vasculature: an emerging concept in antiangiogenic therapy. Science. 2005; 307(5706): 58-62, doi: 10.1126/science.1104819, indexed in Pubmed: 15637262.

6. Weidner N. Intratumor microvessel density as a prognostic factor in cancer. Am J Pathol. 1995; 147(1): 9-19, indexed in Pubmed: 7541613.

7. Gómez-Raposo C, Mendiola M, Barriuso J, et al. Angiogenesis and ovarian cancer. Clin Transl Oncol. 2009; 11(9): 564-571, indexed in Pubmed: 19775995.

8. Matsuda $Y$, Hagio M, Ishiwata T. Nestin: a novel angiogenesis marker and possible target for tumor angiogenesis. World J Gastroenterol. 2013; 19(1): 42-48, doi: 10.3748/wjg.v19.i1.42, indexed in Pubmed: 23326161.

9. Mokrý J, Cízková D, Filip S, et al. Nestin expression by newly formed human blood vessels. Stem Cells Dev. 2004; 13(6): 658-664, doi: 10.1089/scd.2004.13.658, indexed in Pubmed: 15684833.

10. Aihara M, Sugawara Ki, Torii S, et al. Angiogenic endothelium-specific nestin expression is enhanced by the first intron of the nestin gene. Lab Invest. 2004; 84(12): 1581-1592, doi: 10.1038/labinvest.3700186, indexed in Pubmed: 15502861.

11. He QZ, Luo XZ, Zhou Q, et al. Expression of nestin in ovarian serous cancer and its clinicopathologic significance. Eur Rev Med Pharmacol Sci. 2013; 17(21): 2896-2901, indexed in Pubmed: 24254558.

12. Zhou N, Wu X, Yang Bo, et al. Stem cell characteristics of dormant cells and cisplatininduced effects on the stemness of epithelial ovarian cancer cells. Mol Med Rep. 2014; 10(5): 2495-2504, doi: 10.3892/mmr.2014.2483, indexed in Pubmed: 25119644.

13. Klein $\mathrm{D}$, Meissner $\mathrm{N}$, Kleff $\mathrm{V}$, et al. Nestin(+) tissue-resident multipotent stem cells contribute to tumor progression by differentiating into pericytes and smooth muscle cells resulting in blood vessel remodeling. Front Oncol. 2014; 4: 169, doi: 10.3389/fonc.2014.00169, indexed in Pubmed: 25019063.

14. Qin $Q$, Sun $Y$, Fei $M$, et al. Expression of putative stem marker nestin and CD133 in advanced serous ovarian cancer. Neoplasma. 2012; 59(3): 310-315, doi: 10.4149/neo_2012_040, indexed in Pubmed: 22296500.

15. Chen $\mathrm{Z}$, Wang $\mathrm{T}$, Luo $\mathrm{H}$, et al. Expression of nestin in lymph node metastasis and lymphangiogenesis in non-small cell lung cancer patients. Hum Pathol. 2010; 41(5): 737-744, doi: 10.1016/j.humpath.2009.10.018, indexed in Pubmed: 20132963.

16. Hellström $M$, Kalén $M$, Lindahl P, et al. Role of PDGF-B and PDGFR-beta in recruitment of vascular smooth muscle cells and pericytes during embryonic blood vessel formation in the mouse. Development. 1999; 126(14): 3047-3055, indexed in Pubmed: 10375497. 
17. Sá da Bandeira D, Casamitjana J, Crisan M. Pericytes, integral components of adult hematopoietic stem cell niches. Pharmacol Ther. 2017; 171: 104-113, doi: 10.1016/j.pharmthera.2016.11.006, indexed in Pubmed: 27908803.

18. Song S, Ewald AJ, Stallcup W, et al. PDGFRbeta+ perivascular progenitor cells in tumours regulate pericyte differentiation and vascular survival. Nat Cell Biol. 2005; 7(9): 870-879, doi: 10.1038/ncb1288, indexed in Pubmed: 16113679.

19. Paulsson J, Ehnman M, Östman A. PDGF receptors in tumor biology: prognostic and predictive potential. Future Oncol. 2014; 10(9): 1695-1708, doi: 10.2217/fon.14.83, indexed in Pubmed: 25145436.

20. Osman WM, Shash LS, Ahmed NS. Emerging role of nestin as an angiogenesis and cancer stem cell marker in epithelial ovarian cancer: immunohistochemical study. Appl Immunohistochem Mol Morphol.2016 [Epub ahead of print], doi: 10.1097/PAl.0000000000000338, indexed in Pubmed: 26945442.

21. Dong J, Zhao Y, Huang $Q$, et al. Glioma stem/progenitor cells contribute to neovascularization via transdifferentiation. Stem Cell Rev. 2011; 7(1): 141-152, doi: 10.1007/s12015-010-9169-7, indexed in Pubmed: 20697979.
22. Ben-Hamo R, Efroni S. Biomarker robustness reveals the PDGF network as driving disease outcome in ovarian cancer patients in multiple studies. BMC Syst Biol. 2012; 6: 3, doi: 10.1186/1752-0509-6-3, indexed in Pubmed: 22236809

23. Madsen CV, Steffensen KD, Olsen DA, et al. Serum platelet-derived growth factor and fibroblast growth factor in patients with benign and malignant ovarian tumors. Anticancer Res. 2012; 32(9): 3817-3825, indexed in Pubmed: 22993324.

24. Madsen CV, Steffensen KD, Olsen DA, et al. Serial measurements of serum PDGF-AA, PDGF-BB, FGF2, and VEGF in multiresistant ovarian cancer patients treated with bevacizumab. J Ovarian Res. 2012 5(1): 23, doi: 10.1186/1757-2215-5-23, indexed in Pubmed: 22989094.

25. Hosaka K, Yang Y, Seki T, et al. Pericyte-fibroblast transition promotes tumor growth and metastasis. Proc Natl Acad Sci U S A. 2016; 113(38): E5618-E5627, doi: 10.1073/pnas.1608384113, indexed in Pubmed: 27608497.

26. Lu C, Shahzad MMK, Moreno-Smith M, et al. Targeting pericytes with a PDGF-B aptamer in human ovarian carcinoma models. Cancer Biol Ther. 2010; 9(3): 176-182, indexed in Pubmed: 20009575. 